\title{
INTRINSIC CHARACTERIZATIONS OF SOME ADDITIVE FUNCTORS
}

\section{CHARLES E. WATTS}

Our purpose here is to obtain intrinsic functorial characterizations of the functors Hom and $\otimes$ and thus to account in part for the distinguished role played by then in homological algebra. In all that follows, $\Lambda, \Gamma$ are rings with unit, $Z$ the ring of integers. The category of all $\Gamma$ - $\Lambda$-bimodules with $\Gamma$ operating on the left, $\Lambda$ on the right, is denoted by $\Gamma_{\Gamma} \mathbb{N}_{\Lambda}$, the category of left $\Lambda$-modules ( $\Gamma$-modules) by ${ }_{\Delta} \mathfrak{T}\left({ }_{\Gamma} \mathfrak{T}\right)$, etc. All functors are assumed additive. We use throughout the terminology of [1].

THEOREM 1. Let $T$ be a right-exact covariant functor on ${ }_{\Delta} \mathfrak{T H}$ to ${ }_{\Gamma} \mathfrak{T}$ which commutes with direct sums (i.e., is of type $L \Sigma$ ). Then there is an object $C$ in ${ }_{\Gamma} \mathfrak{T l}_{\Lambda}$ and a natural equivalence of functors $\psi: C \otimes_{\Lambda} \approx T$.

Proof. Given an object $A$ of ${ }_{\Delta} \mathfrak{T T}$ and $a \in A$, define $\phi_{a}: \Lambda \rightarrow A$ by $\phi_{a}(\lambda)=\lambda a$. Then $T \phi_{a}: T \Lambda \rightarrow T A$ and we define a function $\psi_{0}^{A}: T \Lambda \times A$ $\rightarrow T A$ by $\psi_{0}^{A}(\tilde{\lambda}, a)=T \phi_{a}(\tilde{\lambda}), \tilde{\lambda} \in T \Lambda, a \in A$. It is easily checked that $\psi_{0}^{\Lambda}: T \Lambda \times \Lambda \rightarrow T \Lambda$ gives $T \Lambda$ the structure of a right $\Lambda$-module, compatible with its left $\Gamma$-module structure. Thus we have made $T \Lambda$ into an object of ${ }_{\Gamma} \mathfrak{T}_{\Lambda} ; T \Lambda$, considered as an object of ${ }_{\Gamma} \mathfrak{T}_{\Delta}$, will be denoted by $C$.

Now for any $A, \psi_{0}^{A}: C \times A \rightarrow T A$ and a simple computation shows that $\psi_{0}^{A}$ is bilinear. Hence $\psi_{0}^{A}$ can be lifted uniquely to a $\Gamma$-homomorphism $\psi^{A}: C \otimes_{\Lambda} A \rightarrow T A$, and the maps $\psi^{A}$ form a natural transformation of functors. Moreover, $\psi^{\Lambda}$ is the natural isomorphism of $C \otimes_{\Delta} \Lambda$ onto $T \Lambda$. Since $T$ and $C \otimes_{\Delta}$ both commute with direct sums, it follows that $\psi^{F}$ is an isomorphism whenever $F$ is a free $\Lambda$-module.

Finally, let $A$ be any $\Lambda$-module; choose an exact sequence

$$
0 \rightarrow R \rightarrow F \rightarrow A \rightarrow 0
$$

with $F$ free. Since $T$ and $C \otimes_{\Delta}$ are right-exact, we have an induced commutative diagram with exact rows

$$
\begin{gathered}
C \otimes R \rightarrow C \otimes F \rightarrow C \otimes A \rightarrow 0 \\
\downarrow \psi^{R} \quad \downarrow \psi^{F} \quad \downarrow \psi^{A} \\
T R \rightarrow T F \rightarrow T A \rightarrow 0
\end{gathered}
$$

Presented to the Society, April 18, 1959, under the title On additive functors; received by the editors April 14, 1959. 
from which it is apparent that $\psi^{A}$ is an epimorphism. Since $A$ was arbitrary, $\psi^{R}$ is an epimorphism. An easy chase about the diagram then reveals that $\psi^{A}$ is a monomorphism, q.e.d.

Now suppose $\Lambda$ is left-Noetherian and let ${ }_{\Lambda} \overline{\mathfrak{T}}$ be the category of finitely-generated left $\Lambda$-modules. Then we can modify the latter part of the above proof by choosing the sequence $0 \rightarrow R \rightarrow F \rightarrow A \rightarrow 0$ so that $F$, and therefore $R$, is finitely generated. Since every functor commutes with finite direct sums, we get

THEOREM 2. If $\Lambda$ is left-Noetherian and if $T$ is any right-exact covariant functor on ${ }_{\Lambda} \overline{\mathfrak{T C}}$ to ${ }_{\Gamma} \mathfrak{T}$, then there is an object $C$ of ${ }_{\Gamma} \mathfrak{M}_{\Lambda}$ and a natural equivalence of functors $\psi: C \otimes_{\Lambda} \approx T$.

For example, if $\Lambda$ is left-Noetherian and $A$ is a left $\Lambda$-module with $\operatorname{dim} A \leqq n$, then $\operatorname{Ext}_{\Lambda}^{n}(A, B) \approx \operatorname{Ext}_{\Lambda}^{n}(A, \Lambda) \otimes_{\Lambda} B$ for each finitelygenerated $B$.

THEOREM 3. Let $T$ be any left-exact contravariant functor on ${ }_{\Lambda} \mathrm{TT}$ to ${ }_{\Gamma} \mathfrak{T}$ which converts direct sums into direct products (i.e., is of type $R \Pi$ ). Then there is a left $\Lambda$ - $\Gamma$-bimodule $C$ and a natural equivalence of functors $\psi: T \approx \operatorname{Hom}_{\Lambda}(, C)$.

The proof of Theorem 3 is completely analogous to that of Theorem 1 , and is omitted. It can be varied to provide an analogue of Theorem 2. These theorems are crucially dependent on the possibility of representing an arbitrary module as a quotient module of a direct sum of copies of $\Lambda$. To characterize the Hom functor in the covariant variable, we first establish the existence of a construction dual to this.

Definitions. If $A$ is a module and $X$ any set, the module $A^{X}$ is defined to be the direct product of copies of $A$ indexed by $X$, or equivalently the set of all functions from $X$ to $A$ under pointwise addition and scalar multiplication. If $A$ and $V$ are two left modules, the evaluation $m a p^{1}$ for $A$ and $V$ is the homomorphism

$$
\alpha: A \rightarrow V^{\text {Hom }(A, V)}
$$

given by $\alpha a(f)=f(a)$. A left module $V$ is distinguishing provided (i) $V$ is injective and (ii) for each left module $A$, the evaluation map for $A$ and $V$ is a monomorphism. (Condition (ii) can be restated: given any nonzero element $a$ of a left module $A$, there exists $f: A \rightarrow V$ such that $f(a) \neq 0$.)

Lemma 4. For every ring $\Lambda$, there exists a distinguishing left $\Lambda$-module.

Proof. Let $V^{\prime}$ be the direct product of all the modules $\Lambda / I, I$ a left

1 This terminology is due to J. L. Kelley. 
ideal of $\Lambda$. Let $V$ be injective, $V \supset V^{\prime}$. If $0 \neq a \in A$, there is a natural map $f^{\prime}: \Lambda a \rightarrow \Lambda / I_{a}$ such that $f^{\prime} a \neq 0$, where $\Lambda a$ is the submodule of $A$ generated by $a$ and $I_{a}$ is the annihilator ideal of $a$. Since $V$ is injective, $f^{\prime}$ can be extended to a map $f: A \rightarrow V$ with $f a \neq 0$.

We remark that the above construction is highly inefficient; if $\Lambda=Z$, then we can take for $V$ the group of rationals mod one.

LEMma 5. Let $T$ be a functor from ${ }_{\Lambda} \mathfrak{T T}$ to ${ }_{\Gamma} \mathfrak{T C}$ which commutes with inverse limits (i.e., is of type $\left.R \Pi^{*}\right)$. Then $T$ commutes with direct products.

For an arbitrary direct product is the inverse limit of finite direct products, and every additive functor commutes with finite direct products.

THEOREM 6. Let $T$ be a covariant left-exact functor from ${ }_{\Lambda} \mathfrak{T}$ to ${ }_{z} \mathfrak{T C}$ which commutes with inverse limits. Then there exists a left $\Lambda$-module $C$ and a natural equivalence of functors $\psi: \operatorname{Hom}_{\Lambda}(C,) \approx T$.

Proof. By Lemma 4, we choose a distinguishing left $\Lambda$-module $V$ and set $C^{\prime}=V^{T V}$. Let $e \in T V^{T V}$ be the identity function; we regard $e$ as a member of $T C^{\prime}$ by means of Lemma 5 .

For each left $\Lambda$-module $A$, we define $\eta_{A}: \operatorname{Hom}\left(C^{\prime}, A\right) \rightarrow T A$ by

$$
\eta_{A}(f)=T f(e), \quad f \in \operatorname{Hom}\left(C^{\prime}, A\right) .
$$

The maps $\eta_{A}$ yield a natural transformation $\operatorname{Hom}\left(C^{\prime},\right) \rightarrow T$. We claim that $\eta_{V}$ maps $\operatorname{Hom}\left(C^{\prime}, V\right)$ onto $T V$. For if $v \in T V$ and $f$ is the $v$ th coordinate projection of $C^{\prime}=V^{T V}$ onto $V$, then $\eta_{V}(f)=T f(e)=v$, because $T$ is of type $R \Pi$ by Lemma 5 .

We next describe the kernel of $\eta_{V}$. If $M$ is any submodule of $C^{\prime}$, we shall identify $T M$ with the submodule $\operatorname{Im} T i$ of $T C^{\prime}$, where $i: M \rightarrow C^{\prime}$ is the inclusion; this is possible because $T$ is left-exact. Let $C$ be the intersection of all submodules $M$ of $C^{\prime}$ such that $e \in T M$. Now the family of such submodules $M$ together with all their inclusion maps make up an inverse limit system, whose inverse limit is $C$. Since $T$ commutes with inverse limits and preserves inclusions, it follows that $T C$ is the intersection of the submodules $T M$ of $T C^{\prime}$. Hence, in particular, $e \in T C$. It follows that if $\eta_{V}(f)=T f(e)=0$, then Ker $f \supset C$, and conversely, if $\operatorname{Ker} f \supset C$, then $e \in T \operatorname{Ker} f=\operatorname{Ker} T f$, so $\eta_{V}(f)=0$. Thus Ker $\eta_{V} \approx \operatorname{Hom}\left(C^{\prime} / C, V\right)$.

Since $e \in T C$, we can define a natural transformation $\psi: \operatorname{Hom}_{\Lambda}(C$, $\rightarrow T$ by

$$
\psi_{A}(f)=T f(e), \quad f \in \operatorname{Hom}(C, A) .
$$


The exact sequence $0 \rightarrow C \rightarrow C^{\prime} \rightarrow C^{\prime} / C \rightarrow 0$ induces an exact sequence

$$
0 \rightarrow \operatorname{Hom}\left(C^{\prime} / C, V\right) \rightarrow \operatorname{Hom}\left(C^{\prime}, V\right) \rightarrow \operatorname{Hom}(C, V) \rightarrow 0
$$

because $V$ is injective. On the other hand, we have shown that the sequence

$$
0 \rightarrow \operatorname{Hom}\left(C^{\prime} / C, V\right) \rightarrow \operatorname{Hom}\left(C^{\prime}, V\right) \stackrel{\eta_{V}}{\rightarrow} T V \rightarrow 0
$$

is exact. Hence $\operatorname{Hom}(C, V) \approx T V$ and it is easy to see that $\psi_{V}$ is the natural isomorphism arising from the last two sequences.

From here on, the proof proceeds much like that of Theorem 1. Let $A$ be any left $\Lambda$-module. The exact sequence

$$
0 \rightarrow A \stackrel{\alpha}{\rightarrow} V^{\mathrm{Hom}(A, V)} \rightarrow \text { Coker } \alpha \rightarrow 0,
$$

where $\alpha$ is the evaluation map, yields a commutative diagram

$$
\begin{array}{ccc}
0 \rightarrow \operatorname{Hom}(C, A) \rightarrow \operatorname{Hom}(C, V)^{\text {Hom }(A, V)} & \rightarrow \text { Hom }(C, \text { Coker } \alpha) \\
\downarrow & \downarrow & \downarrow \\
0 \longrightarrow T & \longrightarrow
\end{array}
$$

in which the central vertical arrow is an isomorphism. We prove as before that each $\psi_{A}$ is an isomorphism, so that $\psi$ is in fact a natural equivalence, q.e.d.

\section{REFERENCE}

1. H. Cartan and S. Eilenberg, Homological algebra, Princeton, 1956.

The University of Chicago 